\title{
Déterminants d'adoption des techniques de production et protection intégrées pour un maraîchage durable à Lubumbashi, République démocratique du Congo
}

\author{
Arsene Mushagalusa Balasha* et Jules Nkulu Mwine Fyama \\ Unité de Recherche en Économie et Développement Agricoles, Faculté des Sciences Agronomiques, Université de Lubumbashi, \\ PO Box 1825, Lubumbashi, République démocratique du Congo
}

\begin{abstract}
Résumé - La production maraîchère est une filière importante de l'agriculture urbaine et périurbaine en Afrique. En République démocratique du Congo, un projet d'horticulture urbaine et périurbaine a été mis en place avec l'appui technique de l'Organisation des Nations Unies pour l'alimentation et l'agriculture pour vulgariser des techniques de production et protection intégrées. Les techniques promues comprenaient l'usage des fertilisants naturels, des extraits de plantes à effet insecticide, des produits naturels de lutte contre les ravageurs, les associations et les rotations culturales, l'usage raisonné des pesticides en dernier recours, ainsi que l'utilisation de variétés résistantes aux maladies. Le modèle Logit a permis d'analyser les données d'enquête d'un échantillon de 246 maraîchers à Lubumbashi entre mars et juin 2016 pour identifier les facteurs favorisant l'adoption des techniques promues. L'appartenance à une association de maraîchers et la formation reçue en maraîchage sont deux facteurs clés qui influencent positivement et significativement l'adoption des techniques de production et protection intégrées, respectivement de 3,5 à 7 fois plus que les agriculteurs n'appartenant pas à une association ou n'ayant pas reçu de formation. Ces résultats suggèrent que la participation des agriculteurs à des ateliers de formation et à des groupes de discussion au travers de champs-écoles peut servir de catalyseur pour accroître l'adoption de techniques innovantes en maraîchage.
\end{abstract}

Mots clés : agriculture urbaine / production intégrée / maraîchage / adoption / innovations

\begin{abstract}
Factors influencing the adoption of integrated production techniques for a sustainable vegetable production in Lubumbashi, Democratic Republic of Congo. Vegetable production is an important sector of urban and periurban agriculture in Africa. In the Democratic Republic of Congo, an horticulture development project was set up between 2000 and 2012 with the technical support of the Food and Agriculture Organization of the United Nations to disseminate integrated production and protection techniques. The techniques promoted included the use of natural fertilizers, seeds resistant to pests, plant extracts with insecticidal effect, natural pest control products, crop associations and rotations, as well as the rational use of pesticides. The Logit model was used to analyze data from a sample of 246 vegetable farmers surveyed between March and June 2016 in Lubumbashi, to identify the factors influencing the adoption of the techniques promoted. Membership in a farmers' association and training in vegetable production appear to be two key factors significantly and positively influencing the adoption of integrated production and protection techniques among farmers, of 3.5 up to 7 times respectively compared to farmers who did not belong to any farmers' group and have never been trained. These results suggest that participation of farmers in training workshops and discussion groups through field schools can serve as catalysts to increase the adoption of innovative techniques in market gardening.
\end{abstract}

Keywords: urban agriculture / integrated production / market gardening / adoption / innovation

\footnotetext{
*Auteur correspondant : arsenemushaga@gmail.com;

mushagalusabalasha@Unilu.ac.cd
} 


\section{Introduction}

La ville de Lubumbashi enregistre une croissance démographique rapide accompagnée d'énormes défis socioéconomiques et environnementaux. De 1984 à 2016, sa population est passée de 580000 habitants (Bruneau et Lootens, 1985) à 2097000 habitants (UN, 2016). Le taux annuel de croissance démographique est d'environ $5 \%$ (Useni et al., 2018) et ce phénomène amplifie la pression anthropique sur les ressources naturelles, notamment la terre, les marais et la forêt autour de la ville (Sikuzani et al., 2018). La FAO (2010) indique que, entre 2002 et 2010, la superficie dédiée à l'agriculture urbaine et périurbaine est passée de 100 à 725 hectares, répartis entre de multiples petites exploitations. On estime à plus de 7800 le nombre de ménages impliqués dans la production de légumes, à la fois pour leur propre subsistance et pour l'approvisionnement des marchés locaux (FAO, 2010). Les femmes jouent un rôle important dans la production maraîchère et la vente des récoltes. L'amarante (Amaranthus spp), le chou de Chine (Brassica chinensis L.) et l'épinard bette (Beta vulgaris subsp vulgaris) sont les principaux légumes produits et consommés à Lubumbashi (Mubemba et al., 2014; Balasha et al., 2015).

En milieu périurbain où sont localisés la plupart des sites maraîchers, la production est associée à certaines mauvaises pratiques comme le brûlis, utilisé par bon nombre d'agriculteurs comme moyen rapide de défrichement. Cette pratique prive le sol d'un apport de matière organique et l'expose à l'érosion. À ce problème s'ajoute aussi l'utilisation, non raisonnée et sans équipements de protection, des pesticides de synthèse; cela expose les utilisateurs à d'énormes risques sanitaires et conduit à la destruction de l'entomofaune utile, telle que les abeilles (Balasha, 2017). Au regard de ces problèmes et de l'importance socio-économique que revêt la production de légumes pour alimenter la ville (Tambwe et al., 2011; Tshomba et al., 2015), un projet d'Horticulture urbaine et périurbaine (HUP) a été mis en œuvre entre 2000 et 2012 avec le soutien financier du royaume de Belgique et l'appui technique de l'Organisation des Nations Unies pour l'alimentation et l'agriculture (FAO). L'un des objectifs de ce projet était de promouvoir un maraîchage durable à travers le développement et l'utilisation des techniques de Production et protection intégrées (PPI) (FAO, 2010 ; Mutshail, 2014). Selon la FAO (2012), la PPI consiste à appliquer des pratiques culturales qui peuvent réduire l'incidence des ennemis des cultures. L'usage de produits phytosanitaires est admis en dernier recours, mais leur usage doit être raisonné et la préférence doit être donnée aux produits qui respectent le mieux l'environnement, l'utilisateur, le consommateur et les auxiliaires. Les techniques vulgarisées à Lubumbashi comprenaient, entre autres, l'utilisation des fertilisants naturels, l'usage raisonné des produits chimiques, l'usage d'extraits de plantes à effet insecticide, l'association et la rotation des cultures, ainsi que l'utilisation de variétés résistantes aux maladies (SENAHUP, 2008). Cependant, l'enquête et les observations conduites en 2016 mettent en évidence une faible utilisation des techniques PPI promues, une augmentation de l'usage des pesticides de synthèse (Balasha, 2017) et de mauvaises pratiques de fertilisation qui ne font qu'accélérer la dégradation des sols (Kesonga, 2017).
Plusieurs études suggèrent que les facteurs socio-économiques sont déterminants dans l'adoption des innovations agricoles (Mabah et al., 2013 ; Mwangi et Kariuki, 2015). Certaines de ces études font ressortir que le sexe, l'âge, le niveau d'étude, l'appartenance à une association d'agriculteurs, la formation agricole reçue, la participation à des champs-écoles, la taille de l'exploitation, le revenu, l'expérience, l'accès au crédit ainsi qu'au marché, influencent le choix des agriculteurs pour l'utilisation des innovations agricoles (Blake et al., 2007; Mabah et al., 2013). L'objectif de ce travail est d'identifier les facteurs socio-économiques pouvant servir de leviers dans la promotion des techniques de production et de protection intégrées pour une agriculture urbaine et périurbaine durable.

\section{Matériels et méthodes}

L'étude a été réalisée de mars à juin 2016 dans cinq sites du périmètre maraîcher de la ville de Lubumbashi, au sud-est de la RD Congo: (i) DAIPN Kisanga, Commune Annexe, située à $11^{\circ} 43^{\prime} 007^{\prime}$ ' de latitude Sud, $27^{\circ} 25^{\prime} 66^{\prime}$ 'de longitude Est et à 1207 m d'altitude; (ii) Kilobelobe, Commune de Kampemba, située à $11^{\circ} 40^{\prime} 306^{\prime \prime}$ de latitude Sud, 2730'974' de longitude Est et à $1279 \mathrm{~m}$ d'altitude, (iii) Maendeleo, Commune de Katuba, située à $11^{\circ} 42^{\prime} 615^{\prime \prime}$ de latitude Sud, $27^{\circ} 27^{\prime} 976^{\prime \prime}$ de longitude Est et à $1207 \mathrm{~m}$ d'altitude, (iv) Naviundu, Commune Annexe, située à $11^{\circ} 37^{\prime}$ '825' latitude Sud, 27³1'266' de longitude Est et à $1248 \mathrm{~m}$ d'altitude, et (v) Tingi-Tingi,

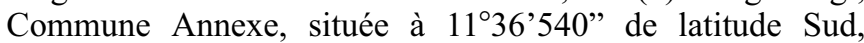
$27^{\circ} 28^{\prime} 433^{\prime \prime}$ de longitude Est et à $1240 \mathrm{~m}$ d'altitude (Fig. 1). Ces sites sont localisés en milieu périurbain et font partie des grands sites maraîchers qui ont bénéficié de l'appui du projet HUP-FAO. Les sites de Naviundu et de Kilobelobe sont parmi les plus anciens et s'étendent respectivement sur 8,5 et 55 hectares, avec une production annuelle estimée à 25000 tonnes de légumes (FAO, 2010). Lubumbashi jouit d'un climat $\mathrm{Cw}$ (climat tempéré chaud avec hiver sec) selon la classification de Köppen ; il est caractérisé par une saison sèche (mai à septembre) et une saison des pluies (novembre à mars), avec un total de $1270 \mathrm{~mm}$ de précipitations par an. La température moyenne annuelle est de $20^{\circ} \mathrm{C}$, avec des minima de $8{ }^{\circ} \mathrm{C}$ et des maxima de $32^{\circ} \mathrm{C}$ (Useni et al., 2018).

Les listes des sites et des maraîchers ont été obtenues auprès des archives du Service national d'horticulture urbaine et périurbaine (SENAHUP), un service technique d'État qui accompagne les maraîchers. À partir de ces listes, 246 producteurs ont été retenus aléatoirement. Les producteurs interrogés n'ont pas été tous bénéficiaires du projet HUP (arrêté en 2012), seuls 102 maraîchers ont reconnu avoir été aidés par ce projet alors que 144 autres n'ont pas bénéficié directement de ce projet. Ces maraîchers sont majoritairement jeunes, de sexe masculin, et échangent sur les techniques de production. En saison sèche, ils produisent les légumes dans les bas-fonds en se servant des cours d'eau et des mares disponibles pour arroser manuellement les cultures. Les sols de ces sites sont contaminés par des métaux lourds provenant essentiellement des activités minières (Mubemba et al., 2014). L'usage des amendements carbonatés et organiques y est vivement recommandé pour réduire les transferts des éléments traces métalliques du sol vers les organes comestibles (Mubemba et al., 2014; Kesonga, 2017). 

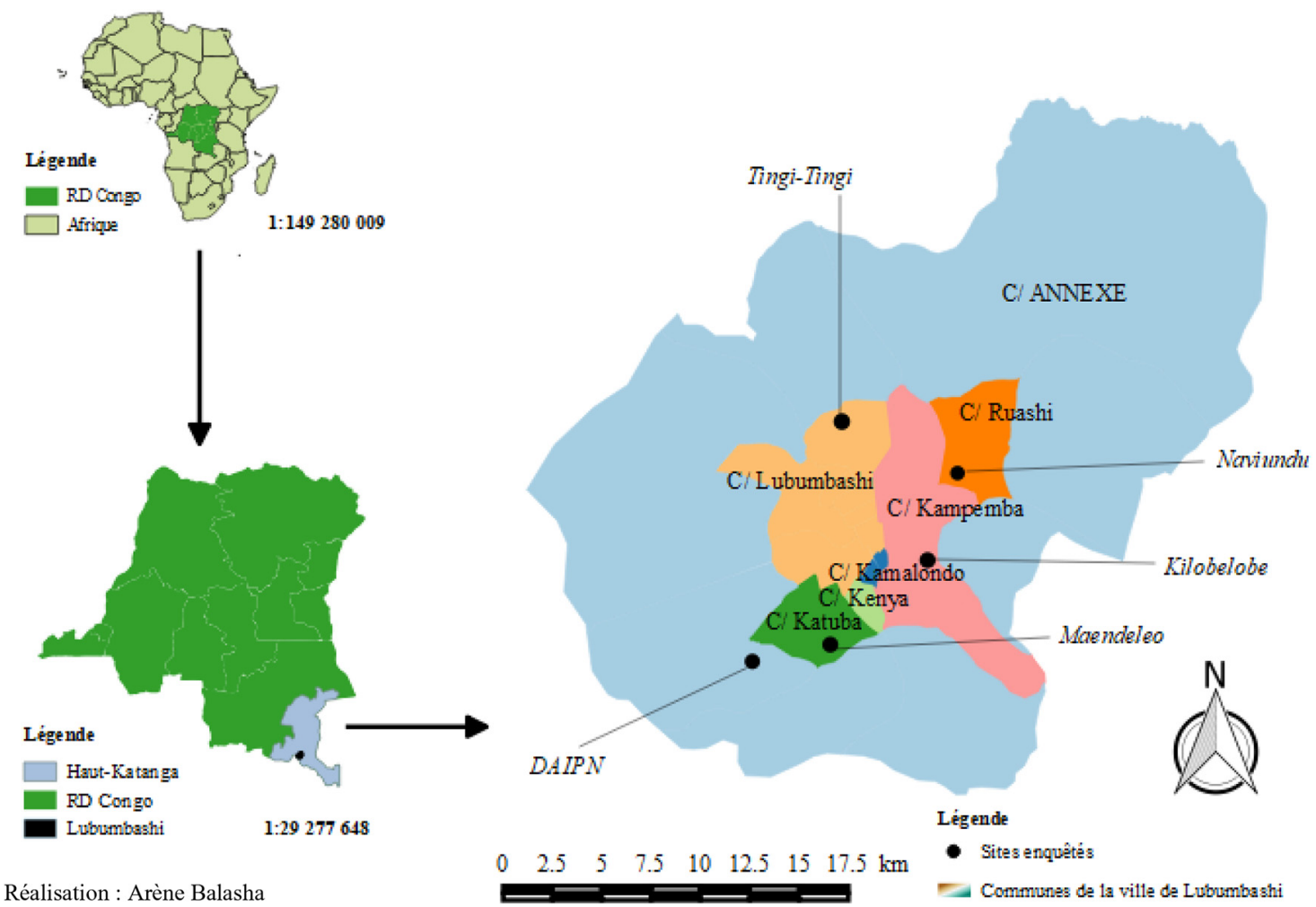

Réalisation : Arène Balasha

Fig. 1. Localisation des 7 communes de la ville de Lubumbashi au sud-est de la RD Congo et des cinq sites maraîchers étudiés.

Fig. 1. Location of the 7 communes of the city of Lubumbashi, southeastern DR Congo and the 5 market garden sites investigated.

\subsection{Collecte des données}

Dans les 5 sites, chaque producteur a été interrogé individuellement pour connaître ses caractéristiques socioéconomiques (genre, âge, niveau d'étude, formation en maraîchage, appartenance à un groupe d'agriculteurs, ancienneté en maraîchage, mode d'accès à la terre, taille de l'exploitation), ainsi que les techniques et intrants utilisés pour produire et protéger les cultures contre les ravageurs et les maladies. Les entretiens avec les producteurs ont été complétés par des observations directes lors des épandages des matières fertilisantes et des produits utilisés pour lutter contre les ravageurs. Les observations directes permettent de mieux comprendre le niveau de connaissance, les attitudes et la perception des agriculteurs vis-à-vis des techniques innovantes vulgarisées (Munyuli et al., 2017).

\subsection{Analyse statistique des données}

Les analyses descriptives effectuées (moyennes, fréquences et pourcentages) résument les données relatives aux caractéristiques socio-économiques. L'adoption est modélisée ici comme un choix entre deux alternatives : adopter (1) ou ne pas adopter (0). Une analyse économétrique par un modèle de régression logistique permet de déterminer les facteurs d'adoption des techniques PPI par les maraîchers. Le modèle Logit (Desjardins, 2005) a été choisi pour les raisons suivantes: (i) le caractère qualitatif et dichotomique de la variable expliquée, à savoir l'adoption ou non des techniques PPI; (ii) ce modèle permet de connaître les facteurs associés à un phénomène en identifiant ceux qui prédisent le mieux le modèle (Desjardins, 2005) ; et (iii) il a été utilisé dans plusieurs études sur l'adoption des innovations agricoles (par exemple Mabah et al., 2013 et Dossa et Miassi, 2018).

Le modèle s'écrit comme suit:

$Y=\mathrm{f}(x, \mathrm{e})$, où $Y$ est la variable dépendante (usage de PPI). Elle prend la valeur 1 si l'agriculteur utilise les techniques préconisées, 0 sinon. $x$ est la matrice des variables susceptibles d'expliquer la variation de $Y$ et e est l'erreur logistique de la distribution.

L'estimation de notre modèle Logit est basée sur la méthode de maximum de vraisemblance. Soit $P i$ la probabilité qu'associe le Logit à l'unité d'enquête,

$$
\begin{aligned}
& P i=F(I i)=\frac{1}{1+e^{-I i}}, \\
& I=\beta 0+\beta 1 X i 1+\beta 2 X i 2+\beta 3 X i 3+\ldots+\beta n X i n,
\end{aligned}
$$

Ii est un vecteur qui représente les caractéristiques de l'unité d'enquête et de la décision de son choix; Les $\beta i$ représentent les coefficients des variables explicatives; les Xin représentent les variables explicatives. Un code binaire a été utilisé pour encoder les variables ( 1 et 0$)$. Les variables utilisées dans le modèle d'adoption des techniques de production et protection intégrées sont décrites dans le tableau 1 . Une valeur de $p<0,05$ a été considérée comme statistiquement significative. 
Tableau 1. Présentation des variables utilisées dans le modèle de régression logistique.

Table 1. Description of variables used in logit model.

\begin{tabular}{ll}
\hline Variables & Description \\
\hline $\begin{array}{l}\text { Variable expliquée } \\
\text { TechPPI } \\
\text { Variables explicatives }\end{array}$ & Techniques PPI prend la valeur 1 si elle a été adoptée, 0 si non \\
Genre & Genre $(1=$ homme $; 0=$ femme $)$ \\
Âge & Tranche âge de l'exploitant $(1=$ jeune $: 17-44$ ans $; 2=$ plus âgé $: 45-67$ ans $)$ \\
Nivetude & Niveau d'étude $(1=$ niveau primaire $; 2=$ niveau secondaire $; 3=$ niveau universitaire $)$ \\
Formation & Formation en maraîchage $(1=$ a suivi une formation relative à la production maraîchère $;$ \\
& $0=$ n'a pas suivi de formation $)$ \\
Association & Membre d'une association des maraîchers $(1=$ est membre $; 0=$ non membre $)$ \\
Expérience & Ancienneté en maraîchage $(1=$ courte $(\leq 5$ ans $) ; 0$ si longue $(>6$ ans $))$ \\
Superficie & Taille de l'exploitation $(1=$ petite $(1-2$ ares $) ; 0$ si large $(\geq 3$ ares $))$ \\
Accesst & Mode d'accès à la terre $(1=$ propriétaire $; 0=$ location $)$ \\
\hline
\end{tabular}

Tableau 2. Caractéristiques socio-économiques des producteurs interrogés $(n=246)$.

Table 2. Socioeconomic characteristics of surveyed vegetable farmers $(n=246)$.

\begin{tabular}{llll}
\hline Variables & & Effectifs & Moyenne ou (\%) \\
\hline Genre & Hommes (\%) & 128 & 52 \\
Âge (années) & Femmes (\%) & 118 & 48 \\
Instruction dominante (secondaire) (\%) & & - & 455 \\
Expérience en maraîchage (années) & & - & 63 \\
Membres d'une association (\%) & & 98 & 9 \\
Formation en maraîchage(\%) & Location & 124 & 40 \\
Mode d'accès à la terre (\%) & Héritage & 111 & 50 \\
& Jouissance temporaire & 98 & 45 \\
Taille de l'exploitation (ares) & & 37 & 40 \\
\hline
\end{tabular}

\section{Résultats}

\subsection{Caractéristiques socio-économiques des répondants}

Bien que les hommes soient un peu plus de la moitié des répondants $(52 \%)$, les femmes jouent un rôle clé dans l'agriculture urbaine et représentent $48 \%$ des maraîchers. L'âge des répondants varie de 17 à 67 ans, et les $2 / 3$ environ ont étudié jusqu'à l'école secondaire (Tab. 2). Les producteurs interrogés ont en moyenne une ancienneté en maraîchage de 9 ans. Ils cultivent de petites surfaces (variant de 1 à 6 ares) obtenues soit par location (45\%), soit par héritage (40\%), soit au titre d'occupation temporaire informelle à titre gracieux $(15 \%)$. Ce dernier mode correspond à l'usage du terrain d'une connaissance durant un temps court (parfois moins d'un an, parfois plus) si le propriétaire est empêché de l'exploiter luimême, ou dans l'attente de la conversion de ce terrain vers une autre activité. Ce même mode concerne aussi les exploitants des terrains publics (à titre d'exemple, autour du campus universitaire de Lubumbashi), où les étudiants maraîchers n'ont pas de contrat précis d'exploitation avec l'université. Les résultats montrent aussi que $40 \%$ des producteurs sont groupés en association de maraîchers. La moitié des producteurs déclarent avoir bénéficié au moins une fois d'une formation en maraîchage.

\subsection{Pratiques actuelles de production et de protection des cultures}

La plupart des producteurs $(78 \%)$ démarrent les activités de production entre mars et avril, parce que c'est la fin de la saison des pluies et une période favorable pour le maraîchage à Lubumbashi, particulièrement pour la culture du chou de Chine pratiquée par tous les maraîchers. Ils ont recours à diverses pratiques de production et protection intégrées (Tab. 3). Tous les répondants $(100 \%)$ confectionnent des plates-bandes et $52 \%$ associent les cultures (chou de Chine et amarante). Les maraîchers ont précisé leurs motivations pour produire sur des plates-bandes : (1) économie d'eau d'arrosage et de fertilisants, (2) inspection et mobilité aisées pour identifier des plants attaqués par les insectes, (3) faciliter la négociation du prix à la vente des produits.

Il faut noter que l'épinard était peu cultivé lors de notre enquête. Certains agriculteurs ont déclaré que l'épinard est 
Tableau 3. Pratiques actuelles de production et de protection des cultures $(n=246)$.

Table 3. Integrated production and pest management practices $(n=246)$.

\begin{tabular}{lll}
\hline Pratiques actuelles & Nombre de répondants & Pourcentage \\
\hline Période de démarrage des activités (mars-avril) & 191 & 78 \\
Culture sur plates-bandes & 246 & 100 \\
Association des cultures (chou et amarante) & 128 & 52 \\
Rotation des cultures & 145 & 59 \\
Usage d'engrais chimique (urée 46\% N, NPK 17-17-17) & 246 \\
Usage de compost (fientes séchées avec plumes, copeaux, divers déchets organiques) & 104 \\
Usage des pesticides chimiques de synthèse & 240 \\
Usage d'un insecticide & 184 \\
Combinaison de deux insecticides & 56 \\
Lutte mécanique (arrachage de plantes malades, destruction manuelle de larves) & 153 \\
Usage de cendres & 193 \\
Macérats à base de piment & 28 \\
Macérats d'ail & 1 & 92 \\
\hline
\end{tabular}

plus sensible aux maladies et que les pertes post-récoltes sont importantes comparativement au chou de Chine.

La totalité des producteurs interrogés utilisent les engrais minéraux (NPK 17-17-17, urée $46 \% \mathrm{~N}$ ), mais seulement $42 \%$ utilisent des fertilisants organiques, constitués de fientes de poule et de copeaux de bois.

Les maraîchers (à 98\%) appliquent principalement deux types d'insecticides de la famille des organophosphorés (m.a. : dichlorvos $100 \mathrm{~g} / \mathrm{L}$ ) et de celle des pyréthrinoides (lambdacyhalothrine à $50 \mathrm{~g} / \mathrm{L}$ ). Ces deux produits sont respectivement connus sous les noms commerciaux de Lava et Karate/ Lamthrin/Lambex (voir Fig. 2), produits importés dont les notices sont uniquement en anglais, alors que les agriculteurs de la région ne parlent que français, kiswahili ou d'autres dialectes locaux. Cela peut se traduire par une incompréhension des consignes de base de sécurité et de mode d'emploi des pesticides. Dans l'espoir d'obtenir plus d'effet sur les ravageurs ciblés, $24 \%$ des maraîchers combinent ces deux insecticides. Aucun des cultivateurs observés pendant la préparation de la bouillie et le traitement des cultures ne portait d'équipements de protection (voir Fig. 3). Pour déterminer les doses de dilution des deux insecticides les plus répandus (à base de dichlorvos et de lambda-cyhalothrine), les maraîchers utilisent des capsules de boissons gazeuses locales dont la contenance est de $7 \mathrm{ml}$ (voir Fig. 3). Ils utilisent deux capsules, soit $14 \mathrm{ml}$ pour $10 \mathrm{~L}$ d'eau, de l'un ou l'autre de ces insecticides pour lutter contre les ravageurs des cultures, notamment les pucerons cendrés du chou (Brevicoryne brassicae), les larves de vers gris (Agrotis ipsilon) et celles de la teigne des choux (Plutella xylostella). Les agriculteurs déclarent que ce sont les dommages causés par ces ravageurs au stade jeune des cultures qui les conduisent à l'application fréquente d'insecticides, et donc à des coûts de production supplémentaires, ainsi qu'à l'achat de jeunes plants pour le remplacement des manquants.

Les pratiques de protection intégrée utilisées comprennent la rotation culturale $(59 \%)$, la pulvérisation de macérats de piment et d'ail sur les cultures comme répulsifs, ainsi que la destruction manuelle des larves de ravageurs $(62 \%)$, réalisée au cours de l'entretien (sarclage, binage) ou lors de l'inspection des parcelles.
La majorité des maraîchers (78\%) épandent les cendres collectées dans leur ménage après combustion du charbon de bois. Il faut signaler que les maraîchers considérent les cendres à la fois comme matières fertilisantes et produits de protection des cultures contre les nuisibles. Deux modes d'épandage ont été observés : (1) épandage à la volée sur les plants en pépinière et sur les cultures jeunes, et (2) épandage localisé au pied de chaque plant en pleine croissance.

\subsection{Facteurs favorisant l'adoption des techniques de production et protection intégrées}

Le nombre d'adoptants de l'ensemble des techniques PPI est inférieur à celui des non adoptants (41\% contre $59 \%)$. Le test de khi-deux effectué montre qu'il y a pas de différence significative entre les hommes $(42 \%)$ et les femmes $(41 \%)$ concernant l'adoption des techniques PPI (Tab. 4). Deux facteurs, parmi les 9 variables soumises à l'analyse, déterminent l'utilisation des techniques PPI, dans l'intervalle de confiance de $95 \%$ (Tab. 5) : l'appartenance à une association de maraîchers et la formation reçue en maraîchage. Ces deux facteurs influencent positivement et significativement l'agriculteur, respectivement de 3,5 à 7 fois, pour utiliser plus les techniques PPI que les agriculteurs n'appartenant pas à une association ou n'ayant pas reçu de formation. Les résultats montrent aussi que l'âge, le sexe, le niveau d'étude, l'expérience en maraîchage, ainsi que la superficie cultivée n'influencent pas significativement $(p>0,05)$ l'adoption des techniques innovantes vulgarisées.

\section{Discussion}

\subsection{Pratiques culturales actuelles et obstacles à I'utilisation des techniques PPI}

Cette étude a montré que seulement $42 \%$ des maraîchers appliquent de la matière organique. Les entretiens avec les agriculteurs sur le terrain ont permis de comprendre les raisons de cette faible utilisation: les maraîchers ont déclaré que l'usage des matières organiques était limité à cause du faible 

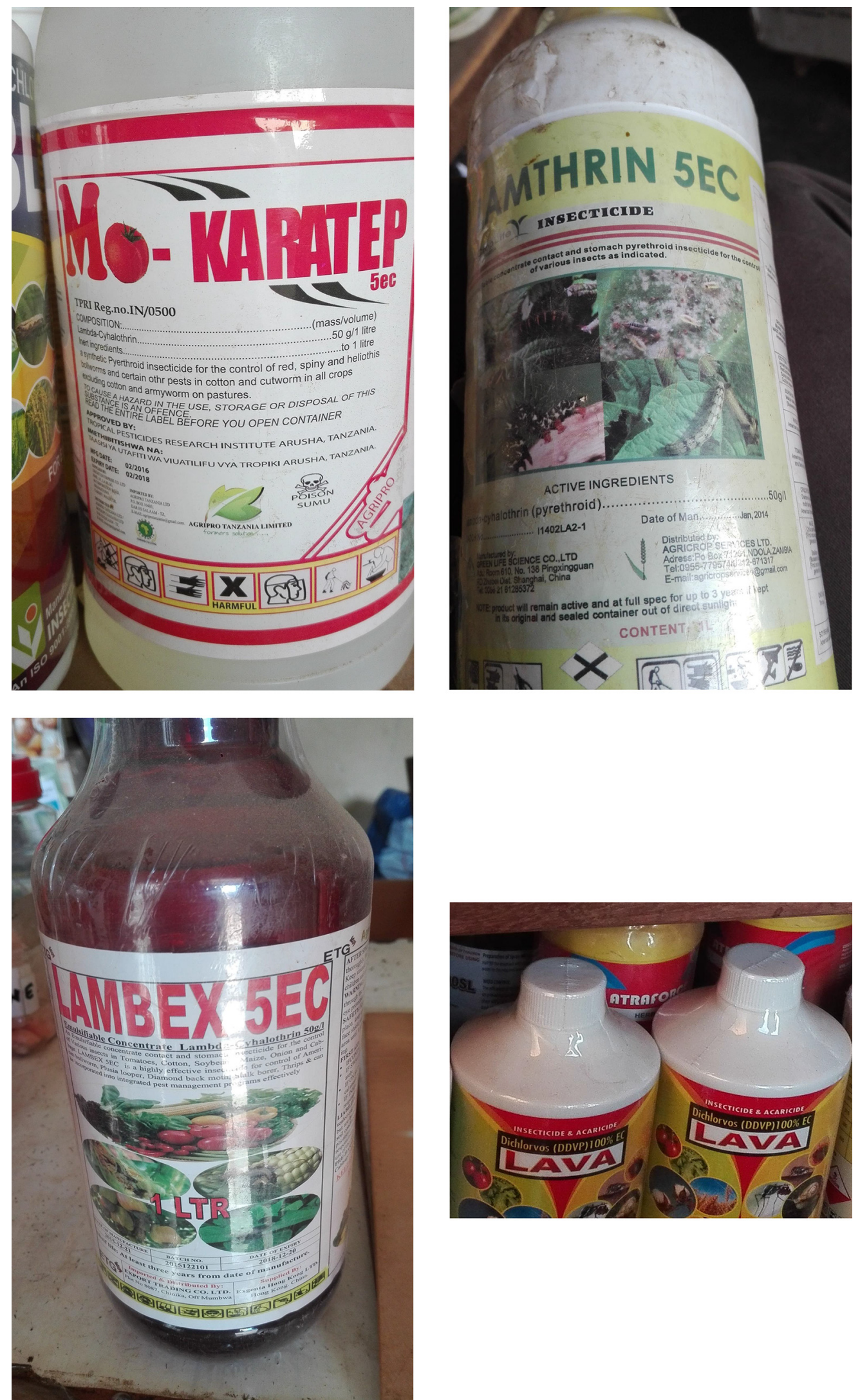

Fig. 2. Trois formulations commerciales de lambda-cyhalothrine et une formulation de dichlorvos utilisées à Lubumbashi (importations de Tanzanie, d'Ouganda et de Zambie).

Fig. 2. Three commercial products containing lambda-cyhalothrin and one containing dichlorvos used in Lubumbashi farms (imported from Tanzania, Uganda and Zambia). 


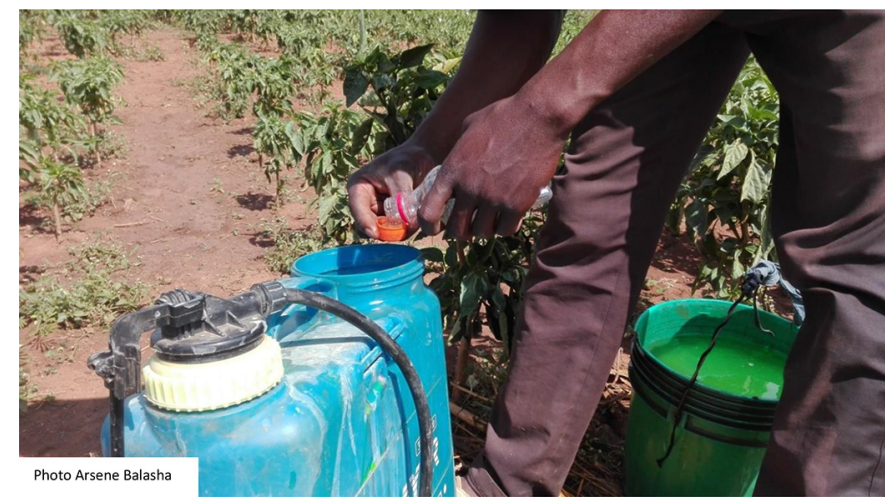

Fig. 3. Un maraîcher, sans équipement de protection, utilise une capsule pour mesurer la dose en vue de préparer la bouillie à pulvériser. Kisanga, Lubumbashi, mai 2017.

Fig. 3. A vegetable farmer preparing a mixture of pesticides, without protection equipement. Kisanga, Lubumbashi, May 2017.

développement de l'élevage en milieu périurbain et des coûts de transport élevés, car les lieux d'approvisionnement sont distants par rapport aux sites maraîchers. Le même constat a été fait par Minengu et al. (2018) à Kinshasa où le prix élevé de la matière organique en milieu périurbain a amené de nombreux maraîchers à appliquer seulement des engrais chimiques. Cette tendance est aussi observée à Lubumbashi où les agriculteurs qui utilisent de la matière organique l'associent avec les engrais minéraux. Cette pratique de combinaison des matières fertilisantes organiques et minérales est recommandée aux agriculteurs pour assurer une gestion intégrée de la fertilité du sol (Bationo et al., 2015; Kesonga, 2017). L'utilisation de la matière organique constitue certes un outil utile pour la restauration et le maintien de la fertilité du sol (Bationo et al., 2015) mais le problème de sa disponibilité en quantité et en qualité est un facteur limitant important en milieu périurbain.

D'autre part, les maraîchers interrogés ont aussi rapporté que les techniques PPI exigent beaucoup de temps en termes de préparation de compost et d'extraits de plantes. À titre d'exemple, ont été évoqués la macération de piment et d'ail ( $100 \mathrm{~g}$ dans $10 \mathrm{~L}$ d'eau) une demi-heure avant le traitement, et le compostage des résidus agricoles et ménagers pendant plusieurs mois. Au Cameroun et en Ouganda, les agriculteurs ont rapporté aussi que les techniques PPI étaient plus consommatrices en main-d'œuvre pour peu d'avantages, comparées à l'usage d'intrants chimiques (Okolle et al., 2016; Ochago, 2018). En plus du problème de la pénibilité de la préparation des produits naturels, Boni et al. (2017) ont montré que la lenteur de leurs effets, leur faible rémanence et leur spectre d'action très réduit, comparé à celui des pesticides chimiques, ont alimenté chez les agriculteurs des doutes sérieux sur leur efficacité.

Après le projet HUP, beaucoup de maraîchers, par manque de motivation et d'accompagnement technique, sont retournés à leurs anciennes pratiques de production, en ignorant les techniques PPI apprises avec le projet. Beaucoup de maraîchers, et surtout des femmes ( $74 \%$ des bénéficiaires), ont été initiés à la fabrication de compost, à la préparation des extraits aqueux de plantes et à d'autres bonnes pratiques agricoles, mais le nombre de ceux continuant à les utiliser a
Tableau 4. Taux d'adoption des techniques de production et protection intégrées par les maraîchers.

Table 4. Adoption rate of integrated production and pest management techniques among farmers.

\begin{tabular}{lll}
\hline Genre & Adoptants & Non adoptants \\
\cline { 2 - 2 } & $n(\%)$ & $n(\%)$ \\
\hline Hommes & $54(42)$ & $74(58)$ \\
Femmes & $48(41)$ & $70(59)$ \\
Total & $102(42$ & $144(59)$ \\
Test & $\chi^{2}=0,58$ & $P=0,81$ \\
\hline
\end{tabular}

progressivement diminué après le projet HUP (Balasha, 2017 ; Balasha et Kesonga, 2019). Le nombre de femmes a d'ailleurs diminué et on est passé de plus de $55 \%$ de femmes en maraîchage à Lubumbashi en 2010 (Tambwe, 2010) à $44 \%$ en 2017 (Balasha, 2017; Kesonga, 2017). Cette diminution de la part des femmes parmi les maraîchers s'explique par diverses raisons : (i) la difficulté de payer les frais de location de terre de plus en plus élevés, variant, en 2015 , de 10 à 135 dollars américains pour une superficie comprise entre 5 à 18 ares (Kasanda et al., 2016); (ii) le lotissement des terres coutumières suivi de leur conversion progressive à d'autres activités, a conduit à l'expulsion d'une proportion importante de femmes ( $47 \%)$ autrefois encadrées par le projet HUP et qui jouissaient, sans contrat précis, d'une bonne partie de ces terres dans la zone périurbaine de Lubumbashi (SENAHUP, 2008); (iii) l'urbanisation rapide de cette zone, qui affecte tous les maraîchers (Sikuzani et al., 2018); (iv) des barrières socioculturelles persistantes qui empêchent les femmes d'hériter du patrimoine foncier ou de le gérer.

Les résultats montrent que, pour contrôler les bioagresseurs, la lutte chimique est privilégiée (98\% des sondés). Les insecticides utilisés par les agriculteurs sont cependant fortement critiqués, car considérés comme à l'origine de sérieux problèmes pour la santé humaine et la biodiversité, et pouvant favoriser l'apparition de résistances chez les ravageurs (Barzman et al., 2015; Damalas et Koutrouba, 2017). Dans une étude antérieure, $76 \%$ des maraîchers interrogés à Lubumbashi sur l'usage des pesticides avaient rapporté le cas d'irritation des yeux et de la peau, et $22 \%$ des sondés avaient mentionné la mort d'abeilles après pulvérisation (Mushagalusa et Kesonga, 2019). La situation reste préoccupante dans cette zone, car la commercialisation et l'usage de pesticides extrêmement toxiques comme le dichlorvos sont encore largement notés, alors que ce dernier est déjà interdit dans plusieurs régions du monde, comme l'Union européenne (European Parliamentary Research Service, 2018) et le Sahel en Afrique de l'Ouest (Rabiou, 2019). Étant très volatile, le dichlorvos est de plus considéré comme peu efficace pour contrôler les larves de vers gris, qui sont le plus destructrices la nuit, alors que, dans les pratiques phytosanitaires de la région, le traitement se déroule vers $9 \mathrm{~h}$ et vers $16 \mathrm{~h}$, pendant que ces larves sont encore dans leurs cachettes souterraines (Mushagalusa et Kesonga, 2019). Même si l'approche PPI n'exclut pas totalement l'usage des intrants chimiques (FAO, 2012), les agriculteurs doivent opérer un 
Tableau 5. Déterminants d'adoption des techniques de production et de protection intégrées.

Table 5. Drivers of adoption of integrated production and pest management techniques.

\begin{tabular}{|c|c|c|c|c|c|c|}
\hline Variables & Coefficient & Erreur St. & $P$-value & $\operatorname{Exp}(B)$ & \multicolumn{2}{|c|}{$95 \%$ IC } \\
\hline Genre & 0,075 & 0,370 & 0,840 & 1,078 & 0,522 & 2,223 \\
\hline Âge & $-0,527$ & 0,354 & 0,136 & 0,590 & 0,295 & 1,181 \\
\hline Niveau d'étude & $-0,468$ & 0,273 & 0,086 & 0,626 & 0,367 & 1,069 \\
\hline Formation & $1,941^{* * *}$ & 0,348 & 0,000 & 6,967 & 3,519 & 13,793 \\
\hline Surface & $-0,518$ & 0,351 & 0,140 & 0,596 & 0,299 & 1,186 \\
\hline Access terre & $-0,011$ & 0,340 & 0,973 & 0,989 & 0,508 & 1,924 \\
\hline Constante & $-1,471$ & 1,595 & 0,356 & 0,230 & & \\
\hline
\end{tabular}

Nombre d'observations $=246 ;-2 \log$ vraisemblance $=231,749 ; \mathrm{R}^{2}$ (Nagelkerke $)=0,457 ;$ Khi-chi-deux $=102,074 ; P=0,000 ; * * *=$ significatif à $1 \%$, IC : intervalle de confiance.

choix judicieux du pesticide à utiliser (efficace, abordable, peu toxique et homologué), sans oublier l'importance des équipements de protection normalement obligatoires.

En vue d'assurer la sécurité des agriculteurs dans un contexte où leur choix de produits phytosanitaires est souvent influencé par les détaillants (Ngowi et al., 2007 ; Mushagalusa et Kesonga, 2019), beaucoup de recherches recommandent à l'autorité compétente (qui a le pouvoir de réguler le marché des produits phytopharmaceutiques) de retirer du marché des pesticides obsolètes dont les propriétés cancérigènes et génotoxiques ont été prouvées (European Parliamentary Research Service, 2018).

L'étude montre que l'association des cultures est pratiquée par un peu plus de la moitié (52\%) des agriculteurs interrogés. Beaucoup d'agriculteurs qui ont opté pour l'association des cultures déclarent que cela permet de réduire la fréquence d'arrosage (une fois par jour au lieu de deux en monoculture), parce que le sol reste plus longtemps humide; les cultures associées sont aussi moins attaquées par les insectes et les adventices sont moins abondantes dans les champs. La stratégie de diversification des cultures est recommandée par le SENAHUP et les chercheurs car elle permet un maintien de la fertilité du sol, favorise la gestion intégrée des bioagresseurs et permet une meilleure sécurité des revenus des producteurs (Bonté, 2010). Malgré les avantages qu'offre cette stratégie, Balasha et Kesonga (2019) ont montré qu'elle n'est pas bienvenue pour beaucoup de maraîchers de Lubumbashi pour trois raisons: (1) les agriculteurs disent que la diversification des cultures maraîchères est difficile à gérer de façon efficace sur de petites surfaces; cela est aussi observé par Marguerie (2011) dans son étude sur la diversification des cultures maraîchères biologiques en France; (2) les agriculteurs disent qu'ils obtiennent des rendements plus élevés en monoculture qu'en association, particulièrement pour la culture d'amarante.

Le mode d'accès à la terre par la location et la jouissance temporaire à titre gracieux constitue un frein pour l'utilisation de la matière organique, comme le reflète le récit de la maraîchère interrogée $\mathrm{N}^{\circ} 22$ du site de Tingi Tingi : «quand on se sacrifie pour amender un terrain de location, c'est travailler parfois pour le prochain contractant ou le propriétaire lui-même, parce que ce n'est pas le même jour qu'on applique le compost que la culture en profite. On n'a pas toujours de garanties de l'exploiter toute l'année». Ce type de réaction montre que le mode d'accès à la terre peut influencer les pratiques de l'agriculteur. Les situations d'incertitude foncière conduisent ainsi les maraîchers à préférer des cultures à cycle court et les fertilisants chimiques.

\subsection{Adoption des techniques des production et protection integrées}

La proportion d'adoptants des techniques de PPI est relativement faible par rapport au nombre de maraîchers interrogés. Cependant, ce taux d'adoption noté à Lubumbashi (42\%) est similaire à celui enregistré par Tey et al. (2012) chez les producteurs de légumes de Malaisie (45\%).

On note aussi que les pratiques des agriculteurs sont souvent similaires. Cela s'explique par le fait que les agriculteurs ont tendance à s'imiter en ce qui concerne le choix des spéculations et des pratiques culturales (Balasha et Kesonga, 2019). Par exemple, au cours de l'enquête, nous avons observé que tous les agriculteurs avaient planté le chou de Chine; or sa monoculture permanente peut favoriser la propagation des ravageurs et la recrudescence des maladies dans les sites maraîchers (Balasha, 2017).

Les résultats de notre étude montrent que le genre n'influence pas significativement l'adoption des techniques PPI. Un constat similaire a été fait au Kenya où Muriithi et al. (2018) ont montré que le sexe n'était pas un facteur décisif dans l'adoption d'une technique innovante (push-pull) de gestion des ravageurs en agriculture familiale. Les observations réalisées dans les sites étudiés à Lubumbashi ont permis de constater que nombreuses femmes pratiquaient l'association des cultures, épandaient les cendres et exprimaient leurs craintes sur l'usage des pesticides de synthèse. Une étude récente dans le périmètre maraîcher de Lubumbashi a révélé que $36 \%$ des femmes maraîchères sont prédisposées à tester sans hésitation des techniques innovantes pour un maraîchage durable (Mushagalusa, 2019). L'étude d'Erbaugh et al. (2003) a montré que les femmes constituent une cible privilégiée pour diffuser les techniques de production intégrée. 
En termes de classe d'âge, les maraîchers dont l'âge se situe entre 45 et 67 ans sont plus réceptifs aux pratiques de PPI que les jeunes (17 à 44 ans). Cela peut être expliqué par leurs savoirs endogènes, les échanges avec leurs pairs et leur longue expérience en agriculture. Le même constat a été fait au Malawi et en Zambie, où Nyirenda et al. (2011) ont montré l'importance de la longue expérience des agriculteurs et de leurs savoirs locaux sur les plantes à effet insecticide. L'étude de Mohammad et al. (2017) en Iran a aussi montré que les producteurs ayant une longue expérience en oléiculture avaient des connaissances plus avancées sur l'identification et la lutte préventive de la mouche de l'olive (Bactrocera oleae). Velay et al. (2001) en Ouganda et Baco et al. (2007) au Bénin, ont aussi mis en évidence le rôle du savoir paysan pour la gestion des bioagresseurs et la conservation de la biodiversité en agriculture familiale.

Nos résultats montrent que l'appartenance à une association de maraîchers et une formation antérieure en maraîchage sont deux facteurs clés qui influencent positivement et significativement l'adoption des techniques de PPI. La représentante des agriculteurs de Maendeleo a souligné que le regroupement des maraîchers en associations les rend plus crédibles vis à vis des organisations locales et internationales (Vision mondiale, FAO, CARITAS), qui les accompagnent alors dans le renforcement de leurs capacités en leur donnant des formations et en leur apportant un appui technique (distribution de semences, arrosoirs, matériels aratoires). Balasha et Kesonga (2019) ont montré que les associations de maraîchers ont permis aux membres d'accroître leur capacité de négociation et de coopération avec des organisations locales et les structures étatiques (SENAHUP, ministère de l'Agriculture et du développement rural). Nos résultats vont dans le même sens que ceux de Mwangi et Kariuki (2015), qui ont montré que l'appartenance à une association paysanne influençait positivement l'adoption de nouvelles technologies agricoles. Cela s'explique par le fait que les agriculteurs d'un groupe social partagent et échangent sur les avantages de l'utilisation des techniques innovantes (Mwangi et Kariuki, 2015). Les maraîchers interrogés ont ainsi confirmé qu'ils discutaient entre eux des problèmes de fertilité du sol, du choix des pesticides et de la période de vente des cultures.

La formation en maraîchage contribue quant à elle à améliorer les connaissances des agriculteurs dans le domaine de la production intégrée et permet d' éveiller leur conscience sur les problèmes liés à l'usage des intrants chimiques (Al-Zyoud, 2014 ; Damalas et Koutrouba, 2017 ; Mohammad et al., 2017). Beaucoup de maraîchers de Tingitingi, de DAIPN et de Kilobelobe nous ont ainsi confirmé que, grâce à des formations sous forme de champs-écoles et des discussions en groupe, ils ont appris à identifier les insectes nuisibles aux cultures et à les distinguer des auxiliaires. Tey et al. (2012) indiquent que l'augmentation de ses connaissances amène l'agriculteur à accepter les pratiques durables qui peuvent entraîner un changement sur son exploitation agricole. Cependant, la volonté des agriculteurs d'utiliser les techniques durables sur le long terme risque d'être mise à mal par l'efficacité à court terme des pesticides et des engrais minéraux. Pour Boni et al. (2017), l'efficacité immédiate des pesticides de synthèse fait oublier aux agriculteurs les risques sanitaires et environnementaux associés à leur utilisation. Cependant, il faut noter qu'au stade actuel, les méthodes alternatives existantes ne peuvent pas remplacer totalement les intrants chimiques; les agriculteurs doivent être formés à les utiliser de manière raisonnée et sécurisée (Balasha, 2017 ; Mushagalusa, 2019). Cela passe, par exemple, par la participation des agriculteurs à des groupes de discussion au travers des champs-écoles et à des ateliers de formation qui peuvent servir de catalyseurs pour l'utilisation des techniques innovantes en agriculture familiale (Kabir et Rainis, 2015).

\section{Conclusion}

La nécessité de protéger les agriculteurs, les consommateurs et les agroécosystèmes des dangers liés à l'emploi non raisonné des intrants chimiques est au cœur de l'exploration et de la vulgarisation des pratiques durables de production agricole, particulièrement en maraîchage. Bien que nos résultats montrent que l'appartenance à une association de maraîchers et la formation en maraîchage influencent significativement et positivement l'adoption des techniques de production et protection intégrées, la réticence et les doutes des maraîchers persistent. Cela s'explique par les avantages immédiats qu'offrent les intrants chimiques (engrais minéraux et pesticides de synthèse) comparativement aux techniques PPI, qui sont plus exigeantes en main-d'œuvre et dont les effets sont lents. Cela pose en particulier un problème pour les nombreux maraîchers urbains et péri-urbains dont la situation foncière est précaire, et qui sont donc contraints de privilégier des solutions de court terme. Malgré cette situation, la sécurité sanitaire des agriculteurs et la durabilité de leurs exploitations devraient faire l'objet d'une plus grande préoccupation des pouvoirs publics et devraient être soutenues par des actions concrètes, comme :

- Retirer du marché des pesticides obsolètes dont les propriétés cancérigènes et génotoxiques ont été prouvées ;

- Renforcer les capacité des maraîchers pour améliorer leur connaissance des ennemis des cultures et des règles d'utilisation des pesticides: préciser et expliquer les techniques de diagnostic et l'importance des équipements de protection ( $c f$. Son et al., 2017);

- Promouvoir des formations sous forme de champs-écoles pour un meilleur apprentissage des techniques de gestion intégrée des bioagresseurs et de la fertilité du sol;

- Conduire des recherches pour évaluer les performances agronomiques et économiques de ces techniques pour préciser leur viabilité technique et économique et apprécier leur impact sur le temps de travail et les revenus des maraîchers.

\section{Références}

Al-Zyoud F. 2014. Adoption range of Integrated Pest Management (IPM) techniques by greenhouse vegetable growers in Jordan. Jordan Journal of Agricultural Sciences 10(3): 504-524.

Baco M, Biaou G, Pinton F, Lescure JP. 2007. Les savoirs paysans traditionnels conservent-ils encore l'agrobiodiversité au Bénin ? Biotechnology, Agronomy, Society and Environment 11(3): 201-210.

Balasha M, Lenga A, Muyeketa M, Kahoma M. 2015. Market Garden produce consumption in Lubumbashi: a comparison between two 
types of Cabbage. International Journal of Innovation and Scientific Research 15(1): 88-94.

Balasha M. 2017. Évaluation des pratiques phytosanitaires en agriculture urbaine et périurbaine a Lubumbashi: état des lieux et perceptions des risques. Mémoire de maîtrise, Gembloux Agro Bio-Tech, 74 p. http://hdl.handle.net/2268.2/3075.

Balasha M, Kesonga N. 2019. Évaluation de la performance économique des exploitations de chou de Chine (Brassica chinensis L.) en maraîchage à Lubumbashi en République Démocratique du Congo. Revue Africaine d'Environnement et d'Agriculture 2(1): 11-19.

Barzman M, Paolo B, Nicholas A, Birch E, Piet B, Silke D, et al. 2015. Eight principles of integrated pest management. Agronomy and Sustainable Development (35): 1199-1215. DOI: 10.1007/ s13593-015-0327-9.

Bationo A, Thomas F, Ken G, Valerie K, Rodney L, Abdoulaye M, et al. 2015. Manuel de gestion integrée de la fertilité des sols. In: Fairhurst T, ed. Consortium Africain pour la Santé des Sols, Nairobi, 169 p.

Blake G, Sandler H, Coli W, Pober D. 2007. An assessment of grower perceptions and factors influencing adoption of IPM in commercial cranberry production. R. Agriculture and Food Systems 22(2): 134144. DOI: $10.1017 / \mathrm{S} 1742170507001664$.

Boni B, Pierre S, Assogba K, Armel M, Alabi T, Verheggen F, et al. 2017. Plantes pesticides et protection des cultures maraîchères en Afrique de l'Ouest (synthèse bibliographique). Biotechnology, Agronomy, Society and Environment 21(4): 288-304.

Bonté J. 2010. La rotation des cultures dans les systèmes céréaliers biologiques : peut-on combiner performances économiques, agronomiques et environnementales? Première approche d'analyse multicritères. Mémoire de Master, Montpellier Sup Agro, France, 61 p. http://www.itab.asso.fr/downloads/rotab/rotab-memoir-oct2010.pdf.

Bruneau JC, Lootens D. 1985. Dynamiques démographiques des quartiers de Lubumbashi des origines à nos jours. Cahiers d'Outremer 121-149. https://www.persee.fr/doc/caoum_0373-5834_1985_ num_38_150_3140.

Damalas C, Koutrouba D. 2017. Farmers' training on pesticide use is associated with elevated safety behavior. Toxics 5: 19. DOI: 10.3390/toxics5030019.

Desjardins J. 2005. L'analyse de régression logistique. Tutorial in Quantitative Methods for Psychology 1(1): 35-41.

Dossa F, Miassi Y. 2018. Facteurs socio-économiques influençant l'adoption de Coton biologique au Nord-Est du Bénin: cas de la Commune de Kandi. International Journal of Sciences and High Technologies 6(2): 577-584.

Erbaugh M, Donnermeyer J, Amujal M. 2003. The Role of Women in Pest Management Decision Making in Eastern Uganda. Journal of International Agricultural and Extension Education 10(3): 71-80.

European Parliamentary Research Service. 2018. Regulation (EC) $1107 / 2009$ on the placing of plant protection products on the market. Brussels: European Union, 588 p. DOI: 10.2861/632821.

FAO. 2010. Développer des villes plus vertes en République Démocratique du Congo, $35 \mathrm{p}$.

FAO. 2012. La Production et Protection Intégrées appliquée aux cultures maraîchères en Afrique soudano-sahélienne. Radhort, CDH, 158 p. http://www.fao.org/3/a-az732f.pdf.

Kabir M, Rainis R. 2015. Do Farmers not widely adopt environmentally friendly technologies? Lesson from Integrated Pest Management (IPM). Modern Applied Science 9(3): 20082014.

Kasanda M, Mushagalusa B, Kitsali J, Nkulu J, Bogaert J. 2016. Maraîchage périurbain à Lubumbashi : modes d'accès à la terre et gestion des superficies agricoles. International Journal of Innovation and Applied Studies 4(14): 27-36.

Kesonga Nsele M. 2017. Enquête sur l'usage des matières fertilisantes en agriculture urbaine et périurbaine de Lubumbashi, RD Congo. Mémoire de maîtrise, Gembloux Agro Bio-Tech, Belgique, 75 p. http://hdl.handle.net/2268.2/3019.

Mabah T, Havard M, Temple L. 2013. Déterminants socioéconomiques et institutionnels de l'adoption d'innovations techniques concernant la production de maïs à l'ouest du Cameroun. Tropicultura 31(2): 137-142.

Marguerie M. 2011. Diversification des cultures dans les exploitations maraîchères biologiques: conséquences sur la gestions agronomique et commerciale. Cas de la basse vallée de Durance, PACA. Mémoire de fin d'études, Montpellier Sup Agro, France, 72p.

Minengu JDD, Ikonso M, Mawikiya M. 2018. Agriculture familiale dans les zones péri-urbaines de Kinshasa: analyse, enjeux et perspectives (synthèse bibliographique. Revue Africaine d'Environnement et d'Agriculture 1(1): 60-69.

Mohammad S, Damalas C, Ebadattalab M. 2017. Farmers' Technical Knowledge about Integrated Pest Management (IPM) in Olive Production. Agriculture 7: 101. DOI: 10.3390/agriculture7120101.

Mubemba M, Useni S, Nyembo K, Colinet G. 2014. Effets d'amendements carbonatés et organiques sur la culture de deux légumes sur sol contaminé à Lubumbashi (RD Congo). Biotechnology, Agronomy, Society and Environment 18(3): 367-375.

Munyuli T, Kana C, Rubabura D, Kajivunira, et al. 2017. Farmers' perceptions, believes, knowledge and management practices of potato pests in South-Kivu Province, eastern of Democratic Republic of Congo. Open Agriculture 2: 362-385. DOI: 10.1515/ opag-2017-0040.

Mushagalusa B. 2019. Drivers of adoption of integrated pest management among small-scale vegetable farmers in Lubumbashi, DR Congo. American Journal of Rural Development 7(2): 53-59. DOI: 10.12691 /ajrd-7-2-2.

Mushagalusa B, Kesonga N. 2019. Pesticide use practices by Chinese cabbage growers in suburban environment of Lubumbashi (DR Congo): Main pests, costs and risks. Journal of Applied Economics and Policy Analysis 2(1): 56-64. DOI: 10.12691/jaaepa-2-1-8.

Muriithi W, Kassie M., Diiro G. Geoffrey M. 2018. Does gender matter in the adoption of push-pull pest management and other sustainable agricultural practices? Evidence from Western Kenya. Food Security 10(2): 253-272.

Mutshail G. 2014. Aperçu technologique sur l'horticulture urbaine et périurbaine de la RDC-Cas de la ville de Lubumbashi. Acta Horticulturae. DOI: 0.17660/ActaHortic.2014.1021.201.

Mwangi M, Kariuki S. 2015. Factors Determining adoption of new Agricultural technology by smallholder farmers in developing countries. Journal of Economics and Sustainable Development 6 (5): 208-2016.

Ngowi F, Mbise T, Ijani M, London L, Ajayi C. 2007. Smallholder Vegetable Farmers in Northern Tanzania: Pesticides use practices, perceptions, cost and health effects. Crop Protection (26): 1617-24.

Nyirenda S, Sileshi G, Belmain S, et al. 2011. Farmers' ethnoecological knowledge of vegetable pests and pesticidal plant use in Malawi and Zambia. African Journal Agricultural Research 6: $1525-1537$.

Ochago R. 2018. Gender and pest management: constraints to integrated pest management uptake among smallholder coffee farmers in Uganda. Cogent Food and Agriculture (4): 1-20.

Okolle NJ, Afari-Sefa V, Bidogeza JC, Precillia I, Ngome F. 2016. An evaluation of smallholder farmers' knowledge, perceptions, choices and gender perspectives in vegetable pests and diseases 
control practices in the humid tropics of Cameroon. International Journal of Pest Management 62(3): 165-74.

Rabiou I. 2019. Liste des produits insecticides et acaricides en vente ou utilisés au Niger en 2019. Réseau National des Chambres d'Agriculture du Niger, $15 \mathrm{p}$.

SENAHUP. 2008. Projet d'appui au développement de l'horticulture urbaine et périurbaine à Lubumbashi. www.fao.org/3/ak159f/ ak159f20.pdf.

Sikuzani Y, Cabala K, Halleux, JM, Bogaert, J, Munyemba K. 2018. Caractérisation de la croissance spatiale urbaine de la ville de Lubumbashi (Haut-Katanga, RD Congo) entre 1989 et 2014. Tropicultura 38(1): 98-108.

Son D, Somda I, Legreve A, Schiffers B. 2017. Pratiques phytosanitaires des producteurs de tomates du Burkina Faso et risques pour la santé et l'environnement. Cahiers Agricultures 26: 25005. DOI: $10.1051 /$ cagri/2017010.

Tambwe N. 2010. Urban agriculture and food security in the city of Lubumbashi (DRC). Doctoral dissertation, University of the Witwatersrand, 306 p. http://hdl.handle.net/10539/8176.

Tambwe N, Rudolph M, Greenstein R. 2011. Instead of Begging, I Farm to Feed My Children: Urban Agriculture - An Alternative to Copper and Cobalt in Lubumbashi. The Journal of the International African Institute 81: 391-412.
Tey Y, Elton L, Johan B, Amin M, Jay C, Alias R, et al. 2012. Adoption rate of sustainable agricultural practices: A focus on Malaysia's vegetable sector for research implications. African Journal of Agricultural Research 7(19): 2901-2909.

Tshomba J, Nyembo M, Ntumba N, Mushagalusa B, Muyambo M, Nkulu M. 2015. Le maraîchage et ses fonctions dans le contexte socio-économique de Lubumbashi en RD Congo. International Journal of Innovation and Applied Studies 11(2). 291-302.

UN Department of Economic and Social Affairs, Population Division. 2016. The World's Cities in 2016-Data Booklet (ST/ESA/ SER.A/ 392).

Useni S, Marie A, Mahy G, Cabala K, Malaisse F, Munyemba F, et al. 2018. Interprétation paysagère du processus d'urbanisation à Lubumbashi: dynamique de la structure spatiale et suivi des indicateurs écologiques entre 2002 et 2008. In Bogaert J, Colinet G, Mahy G, eds. Anthropisation des paysages katangais. Gembloux, Belgique : Presses Universitaires de Liège-Agronomie, pp. 281296.

Velay F, Baudoin JP, Mergeai G. 2001. Caractérisation du savoir paysan sur les insectes nuisibles du pois d'Angole (Cajanus cajan (L) Millsp) dans le Nord de l'Ouganda. Biotechnology, Agronomy and Society and Environment 5(2): 105-114.

Citation de l'article : Mushagalusa Balasha A, Nkulu Mwine Fyama J. 2020. Déterminants d'adoption des techniques de production et protection intégrées pour un maraîchage durable à Lubumbashi, République démocratique du Congo. Cah. Agric. $29: 13$. 